This item was submitted to Loughborough's Research Repository by the author.

Items in Figshare are protected by copyright, with all rights reserved, unless otherwise indicated.

\title{
PC-RED for IPv6: algorithm and performance analysis
}

PLEASE CITE THE PUBLISHED VERSION

PUBLISHER

(C) IEEE

VERSION

VoR (Version of Record)

LICENCE

CC BY-NC-ND 4.0

REPOSITORY RECORD

Li, Yunqiu, and Shuang-Hua Yang. 2019. "PC-RED for Ipv6: Algorithm and Performance Analysis". figshare. https://hdl.handle.net/2134/4143. 
This item was submitted to Loughborough's Institutional Repository (https://dspace.lboro.ac.uk/) by the author and is made available under the following Creative Commons Licence conditions.

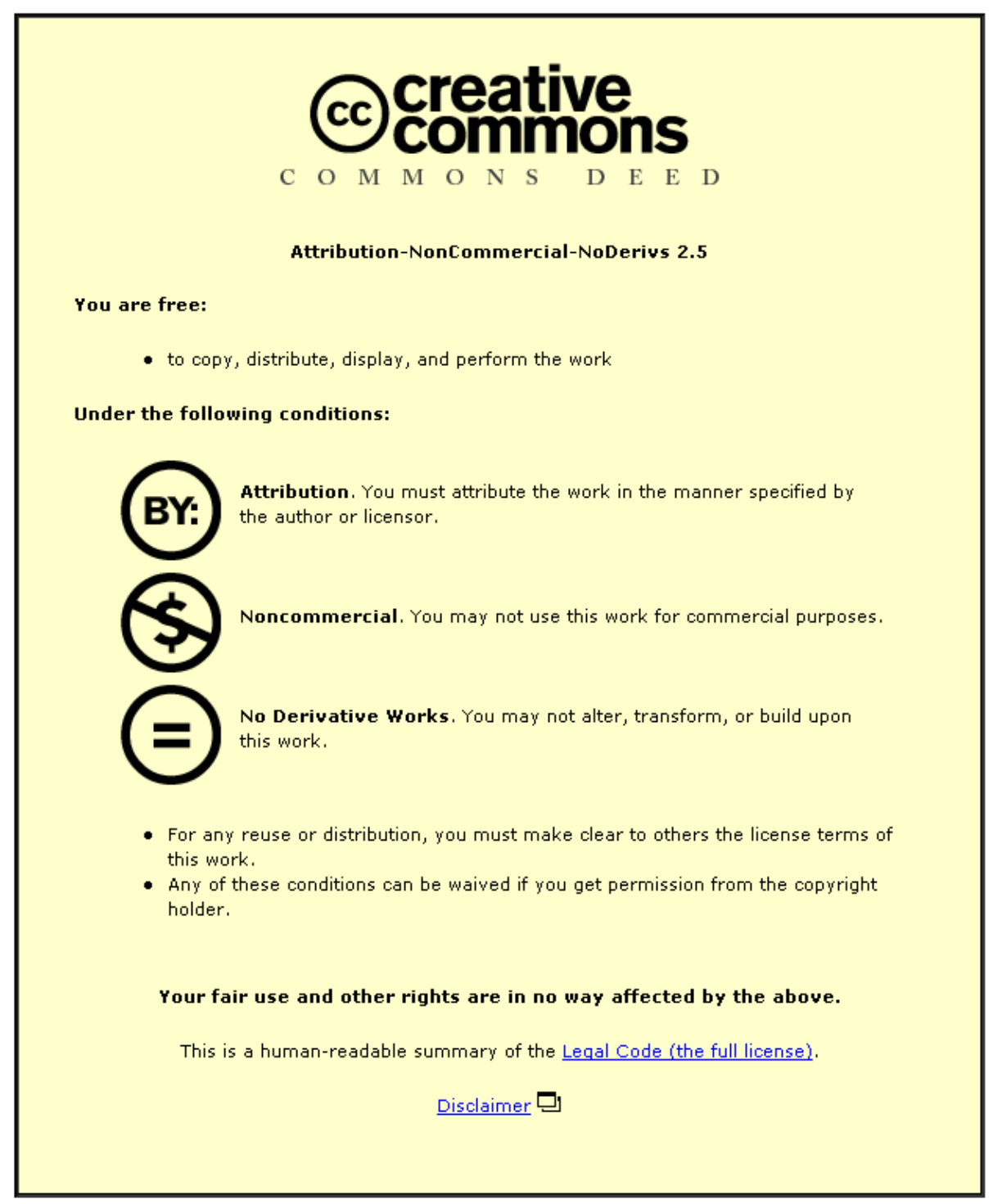

For the full text of this licence, please go to: http://creativecommons.org/licenses/by-nc-nd/2.5/ 


\title{
PC-RED for IPv6: Algorithm and Performance Analysis
}

\author{
Yunqiu LI, Shuang-Hua YANG \\ Computer Science Department, Loughborough University, UK \\ Emails: \{Y.LI3, S.H.YANG\}@lboro.ac.uk
}

\begin{abstract}
This paper presents a Priority Checking Random Early Detection (PC-RED) gateway for ensuring the Quality of Service $(\mathrm{QoS})$ of high priority dataflow in IPv6 networks. A bit in the IP header is used in PC-RED to label the current status of the QoS that the dataflow is being treated in, which is determined by the difference between the packet average-dropping rate and the fixed desired limit dropping rate of the dataflow. PC-RED would perform dissimilarly to every dataflow corresponding to the different QoS status throughout congestions. PC-RED has been modeled and the parameter setting has been studied. Simulation result shows remarkable contrast between the High-Priority and Non-Priority dataflow throughput under PC-RED mechanism.
\end{abstract}

\section{INTRODUCTION}

Research on Internet congestion control comprises two main parts: TCP (Transmission control protocol) and AQM (Active Queue Management) [1]. As the most successful AQM, RED (Random Early Detection) has been widely implemented. Much research [2-4] has been done in this area. The growth of Internet urges the Internet Engineering Task Force (IETF) to produce the upgraded Internet Protocol IPv6 to satisfy the large demands of IP addresses. The flow label field, which is a new field added in IPv6 header, is used to facilitate identification of data requiring special handling, such as those involved in real-time applications, etc.

Since the Internet nowadays is expected to provide a distinct service to different users based on value, and to deliver a distinct service based on content accessed, Priority checking RED is developed to ensure the end user with higher priority gets the guaranteed QoS according to their demands by implementing a Priority Checking function in RED.

The rest of the paper is organized as follows. In Section 2, the algorithm of PC-RED is introduced. Section 3 focuses on the PC-RED parameter setting. Simulation results obtained by using Network Simulator 2 (NS2) that verify the PC-RED contribution to guaranteeing some form of QoS to particular end user with high priority is presented in Section 4. Finally, the conclusions are given in Section 5.

\section{PRIORITY CHECKING RANDOM EARLY DETECTION}

The IPv6 packets can be labelled with different priorities, so dataflows with different QoS requirements could be classified and probably stored in separate buffers before they are transmitted into the further hop [5]. But such mechanism has disadvantages as it complicates the implementation using separate buffers for different QoS levels, and there is a high probability that the buffer utilization is inferior throughout the whole transmission. Thus, PC-RED overcomes the underutilization of the queue by using one buffer for multi- dataflows, and a different mechanism to pick which packet to drop when there is congestion.

One bit in the IPv6 header, referred to as QOSA (Quality Of Service Alert), is used to indicate whether the dataflow has been treated worse than was expected. During the transmission, the dataflows have certain levels of packet average-dropping rate $a_{d}$. If $a_{d}$ exceeds the desired limit $L$, QOSA would be set to 1 to notify PC-RED to implement some form of protection.

In the PC-RED router, an extra database is set up referred to as the Priority List. The priority list is a list containing the priority limit and the current status of every data flow passing through the router. The elements of the list are: Data flow ID, Priority limit, Last dropping time, $a_{d}$ and QoS Status.

The working processes of PC-RED are shown in Figure 1. Blocks in grey illustrate the differences compared with the traditional RED. The priority list maintenance function is triggered each time the router receives a packet. When PCRED adds a new entry to the priority list with the dataflow id as the index element, $a_{d}$ would be set to half of $L$ as default, and the status would be set to zero indicating the average dropping rate is currently lower than the desired limit. In case the dataflow is switched to one route due to the transmission failure in the other routes, a higher start point of the average drop rate could help to notify the router with the QoS level more rapidly. If the flow ID is in the list, PC-RED would check the status element in the entry. If the status is one, the QOSA bit would be set to one, indicating this packet is undroppable, before adding it into the queue. While the average queue length exceeds the threshold, instead of randomly picking the victim, PC-RED only picks packets with 0 as the QOSA. And after dropping a packet, the priority list maintenance function is triggered again to update the priority list with the latest average dropping rate.

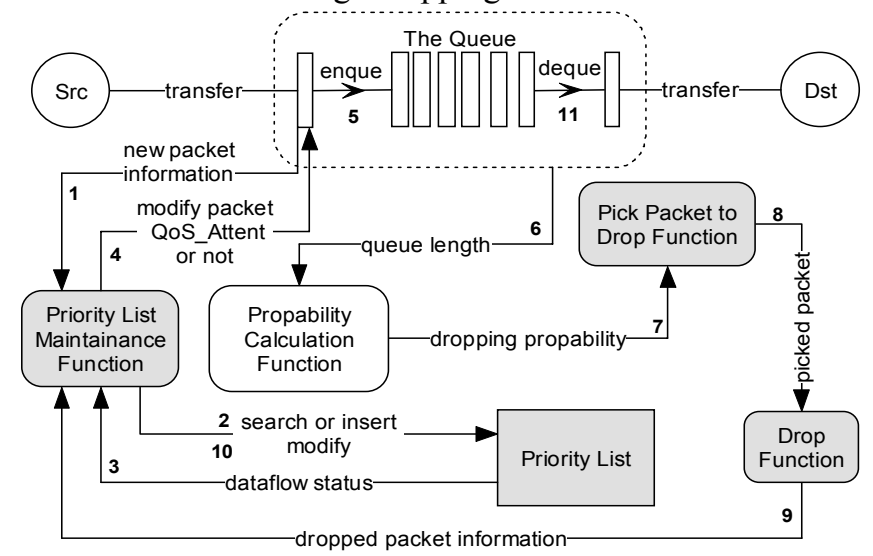

Figure 1 PC-RED Working Processes 
When the average queue length exceeds the threshold, PCRED first randomly picks a packet, and accesses the header to check the QOSA bit. If the QOSA is not zero and it is not the last packet in the queue, it would check to the next packet. Otherwise it will get the packet size, and calculate the latest average dropping rate $a_{d}$, update $a_{d}$ in priority list, and compare it with $L$. If $a_{d}$ exceeds $L$, set the status element to 1 , otherwise, set the status to 0 . Finally it drops the packet and update the last dropping time of the corresponding entry.

In RED, the dropping probability $p(t)$ calculated from the average queue length $q(t)$ is regarded as an overall dropping probability. However, when breaking down the dataflows into high-priority ones and non-priority ones, $p(t)$ can be divided into $p_{p}(t)$, the dropping probability of high-priority dataflows, and $p_{n}(t)$, the dropping probability of the non-priority ones. Thus PC-RED can provide different levels of QoS to dataflows with different priorities by manipulating their dropping probabilities respectively.

\section{PC-RED MODELLING}

In [6], a dynamic model of TCP behaviour was developed using fluid-flow and stochastic differential equation analysis. Based on that, [7] has presented a simplified TCP-queue dynamic model.

For a network with TCP dataflows with different priorities, assume the total number of TCP sessions is $N$, including $N_{p}$ TCP sessions with high-priority and $N_{n}$ TCP sessions with non-priority. In $N_{p}$ sessions, $N_{p+}$ of them have the average-dropping rate $a_{d}$ exceeding the desired limits $L$ and $N_{p-}$ of them have the average-dropping rate $a_{d}$ under the desired limits $L$.

Thus, we have

$$
\left\{\begin{array}{l}
N=N_{p}+N_{n} \\
N_{p}=N_{p+}+N_{p-}
\end{array}\right.
$$

Separating the perturbation of the packet dropping probability $\delta p$ into three parts in terms of the dataflow priorities: $\delta p_{p^{+}}$, the perturbation of the packet-dropping probability of high-priority TCP sessions whose averagedropping rates $a_{d}$ have exceeded the desired limits $L, \delta p_{p-}$, the perturbation of the packet-dropping probability of highpriority TCP sessions whose average-dropping rates $a_{d}$ are under the desired limits $L$ and $\delta p_{n}$, the perturbation of the packet-dropping probability of non-priority TCP sessions, the TCP PC-RED Dynamics has three feed-forward channels as shown in Figure 2.

Where $C_{p+}(s)$ : The transfer function between $\delta p$ and $\delta p_{p+}$; $C_{p-}(s)$ : The transfer function between $\delta p$ and $\delta p_{p_{-}}$; $C_{n}(s)$ : The transfer function between $\delta p$ and $\delta p_{n} ; \phi_{p^{+}}+\phi_{p_{-}}+\phi_{n}=\delta ; L_{\text {red }}=\frac{p_{\max }}{\text { max }- \text { min }} ; K=-\frac{\log _{e}(1-\alpha)}{\delta}$; $\max _{h}$ is the maximum threshold; $\min _{t h}$ is the minimum threshold; $\alpha$ is the average queue weight, used to calculate the average queue length; $\delta$ is the Sample time and $p_{\max }$ is the maximum dropping probability.

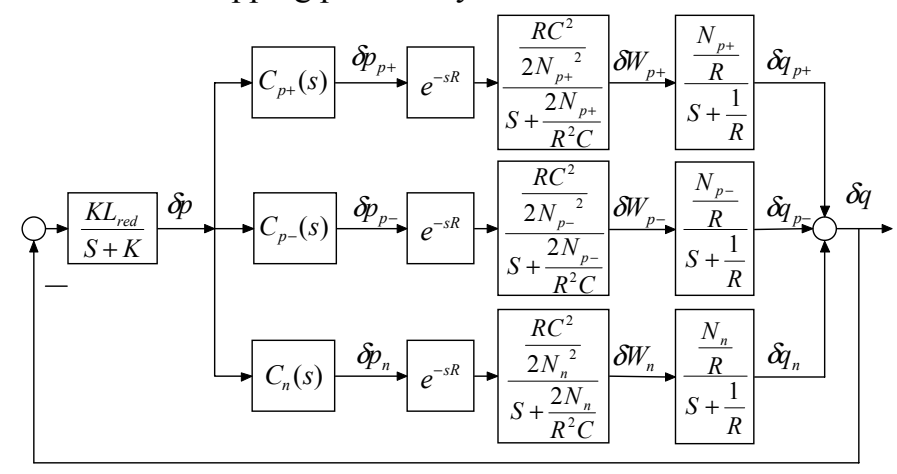

Figure 2 TCP-PCRED Dynamics

According to PC-RED algorithm, we have

$$
C_{p+}(s) \in\left\{\frac{N_{p^{+}}}{N} \times 0, \frac{N_{p^{+}}}{N} \times \frac{1}{q}\right\}=\left\{0, \frac{N_{p+}}{N \times q}\right\}
$$

When $C_{p+}(s)=0$,

$$
\begin{gathered}
C_{p-}(s)=\frac{N_{p-}}{N}+\frac{N_{p+}}{N} \times \frac{N_{p-}}{N-N_{p+}}=\frac{N_{p-}}{N-N_{p+}} \\
C_{n}(s)=\frac{N_{n}}{N}+\frac{N_{p+}}{N} \times \frac{N_{n}}{N-N_{p+}}=\frac{N_{n}}{N-N_{p+}}
\end{gathered}
$$

$$
\text { When } \begin{aligned}
C_{p+}(s) & =\frac{N_{p+}}{N \times q}, \\
C_{p-}(s) & =\frac{N_{p-}}{N}+\left(\frac{N_{p+}}{N}-\frac{N_{p+}}{N \times q}\right) \times \frac{N_{p-}}{N-N_{p+}} \\
C_{n}(s) & =\frac{N_{n}}{N}+\left(\frac{N_{p+}}{N}-\frac{N_{p+}}{N \times q}\right) \times \frac{N_{n}}{N-N_{p+}}
\end{aligned}
$$

\section{PC-RED Parameter SetTing}

The objective of this section is to analyze the model presented in Section 3 thereby guiding the PC-RED parameters setting.

Apart from the traditional RED parameters, such as $\max _{t h}, \min _{t h}, \alpha$ and $p_{\max }$, which have been introduced earlier, the extra pre-defined parameters in PC-RED are $r_{p \max }$ and $w . r_{p \max }$ is the maximum threshold of $r_{p}$, the ratio of the high-priority dataflows to the overall data load $N$, above which the PC-RED has difficulty in guaranteeing high throughput to high-priority dataflows. $w$ is the weight factor which is used to calculate the average dropping rate $a_{d}$ and can be tuned to adjust the responding speed of $a_{d}$ to the dropping action.

\section{A. $r_{p \max }$ and System Stability}

Comprehensively consider the PC-RED algorithm and Figure 2, it is observed that the main objective of implementing PC-RED as an AQM is to guarantee that highpriority dataflow could achieve high-level throughput, in 
terms of manipulating $\delta p_{p+}$ in order to keep $a_{d}$ stable and oscillating around the desired dropping limit $L$ so to control the perturbation of the high-priority dataflow window size $\delta W_{p+}$.

Assume all of the TCP dataflows are with the same set of parameters and the packet losses to dataflow $i$ are described by a Poisson process with time varying rate. Thus at time $t$, from Figure 2, the critical state of the system is the entire $N_{p}$ has become $N_{p+}$ due to the lack of control, the following equation can be observed.

$$
\begin{aligned}
& \delta \dot{W}_{p+}=-\frac{2 N_{p+}}{R^{2} C} \delta W_{p}-\frac{R C^{2}}{2 N_{p+}^{2}} \cdot \frac{N_{p+}}{N \cdot q(t)} \cdot \delta p(t-R) \\
& \approx-\frac{2 r_{p} N}{R^{2} C} \delta W_{p}-\frac{R C^{2} \cdot \delta p(t-R)}{2 r_{p} \cdot N^{2} \cdot q(t)}
\end{aligned}
$$

Where $\delta \dot{W}$ denotes the time-derivative of the perturbation of the window size. Thus, the frequency response of the transfer function could be generated as follow.

$$
L(j \omega)=\frac{L_{r e d} \frac{(R C)^{3}}{4 r_{p}{ }^{2} N^{3} q} e^{-j \omega R}}{\left(\frac{j \omega}{K}+1\right) \cdot\left(\frac{j \omega}{\frac{2 r_{p} N}{R^{2} C}}+1\right) \cdot\left(\frac{j \omega}{\frac{1}{R}}+1\right)}
$$

According to $L(j \omega)$, if $r_{p}$ follows the equation below:

$$
r_{p}<\frac{R C}{2 N}
$$

when set $\omega \in\left[0, \omega_{g}\right], \omega_{g}=\frac{r_{p} N}{5 R^{2} C}$, and the RED parameters setting follows

$$
L_{\text {red }} \frac{(R C)^{3}}{4 r_{p}{ }^{2} N^{3} q} \leq \frac{\omega_{g}}{K}
$$

the close loop system is stable. As we can obtain,

$$
\begin{gathered}
\left|L\left(j \omega_{g}\right)\right|<\frac{L_{\text {red }} \frac{(R C)^{3}}{4 r_{p}{ }^{2} N^{3} q}}{\frac{\omega_{g}}{K}}<1 \\
\angle L\left(j \omega_{g}\right) \geq \angle \frac{L_{\text {red }} \frac{(R C)^{3}}{4 r_{p}{ }^{2} N^{3} q}}{\frac{j \omega_{g}}{K}+1}-\omega_{g} R \geq-90^{\circ}-0.1 \frac{180^{\circ}}{\pi}>-180^{\circ}
\end{gathered}
$$

Thus, from Bode Plots, a stable system is achieved.

Therefore the PC-RED limits $r_{p}$ by embedding an advanced packet scheduling mechanism. When the mechanism detects the percentage of high-priority dataflows $N_{p}$ has exceeded the ideal limit $r_{p \text { max }}$, it would redirect the packet to another path in the network. This algorithm will not be further discussed in the following content.

\section{B. $w$ and System Performance}

Once the $r_{p \max }$ is settled, by setting the parameters following (6), a stable system, relatively to both high-priority

and non-priority dataflows, can therefore been constructed. However, the PC-RED is using the difference value between $a_{d}$ and $L$ to determine the QOSA setting therefore to trigger the special dropping mechanism. The way that $a_{d}$ fluctuating around $L$ is also an important issue as the sensitivity of $a_{d}$ directly impacts on the PC-RED behaviour.

EWMA (Exponentially Weighted Moving Average) is used here as a low-pass filter to calculate the average-dropping rate $a_{d}$. Thus the packet droppings caused by a short-term increase of the average queue size will not result in a significant increase in the average-dropping rate.

If the $a_{d}$ is calculated in every standard dropping time interval $g$, and the packet size is fixed under the circumstances of research simplicity, assuming the dropping occurs when the number of interval is $n$, after $m$ intervals, there is another dropping.

Thus the following equation can be developed:

Where

$$
a_{d(n+m)}=(1-w)^{m} \times a_{d(n)}+w \times x_{(n+m)}
$$

$$
\begin{array}{lr}
m=\frac{t-t_{n}}{g} & \\
t & = \\
t_{n} & = \\
g & = \\
w & =
\end{array}
$$$$
x_{(n+m)}=
$$

timet.

Take one high-priority dataflow for example, based on (7), assuming that the average-dropping rate is initially set to be half of the desired limit $L$, the dropped packet is fixed as $X$ throughout the transmission and there is packet-dropping in every standard interval, thus we have:

$$
a_{d(n)}=f(w, n)=(1-w)^{n} \times \frac{L}{2}-(1-w)^{n} \times X+X
$$

Figure 3 shows the average-dropping rate $a_{d}$ as the function of the weight factor $w$ and the packet dropping counter $n$, when the desired limit $L$ is converted into size and set to be 300 and $X$ is 500 . It is shown that the ascending extent of $a_{d}$ increases evidently to the increase of the weight factor $w$.

If $w$ is too large, then the dataflow would be very sensitive to the packet dropping. Thus the probability of $a_{d}$ exceeding the desired limit would also increase. This might be propitious to the transmission of high-priority dataflows. However, this might also lead to a complete sacrifice of non-priority dataflows' transmission, as in equations (2), (3) and (4), a large $N_{p}$ would result in large $C_{p-}(s)$ and $C_{n}(s)$, which are the factors used to calculate the dropping probability $p_{p+}$ and $p_{n}$. If $w$ is too small, then $a_{d}$ would not be a reasonable reflection 


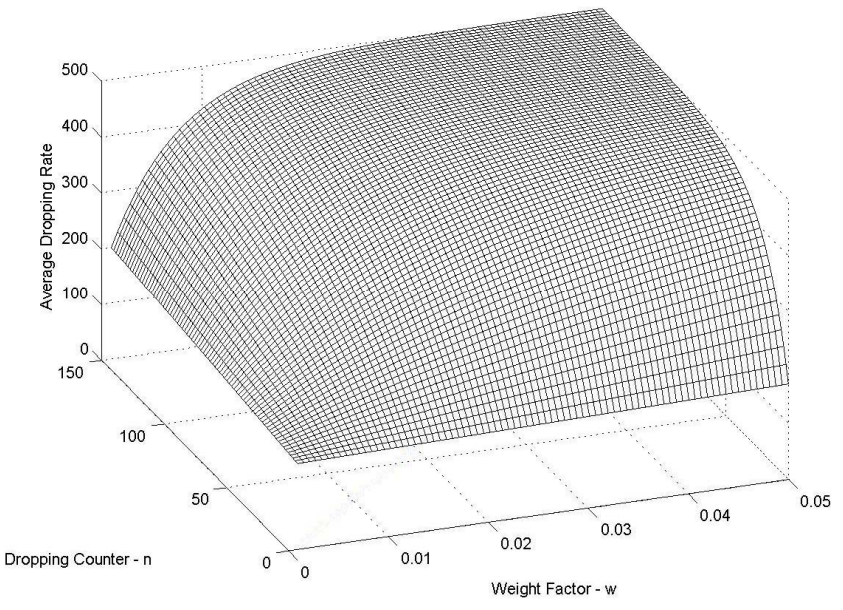

Figure $3 a_{d}$ as the function of the weight factor $w$ and the packet dropping counter $n$

of the current dropping action, as it would respond too slowly to the dropping. In this case, the PC-RED would not be able to detect the current level of QoS the high-priority dataflows are being treated with. In the scenario presented in Figure 3, if the PC-RED is expected to respond the dropping action within 50 to 100 standard intervals, the $w$ should be set in the range of approximate 0.003 to 0.03 .

\section{SIMULATION}

Our simulation is implemented in NS2. The experiment includes the following steps. First, we use traditional RED and PC-RED in a single bottle-link network to analysis the stability of the queue. The Network topology is shown in Figure 4.

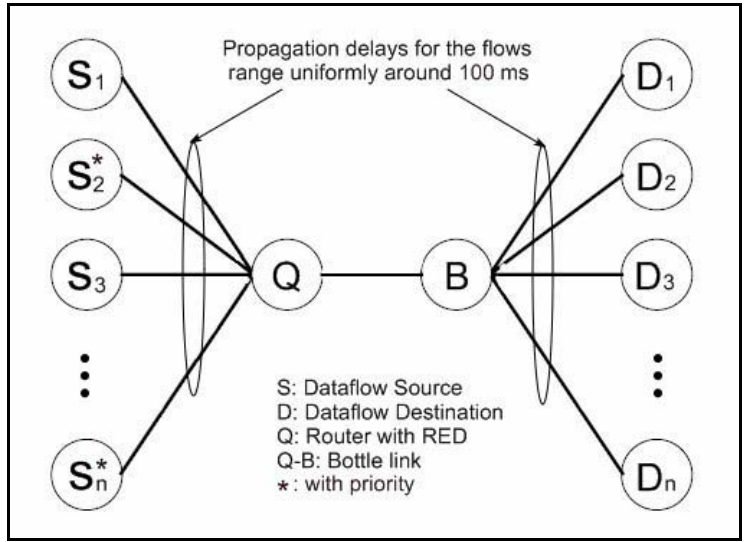

Figure 4 Single Bottle-Link Network Topology

Then we analyse the throughput of one node in two different network topologies. Network 1 is shown in Figure 4 and Network 2 is a mash network shown in Figure 5. Both of them contain certain percentage of high-priority dataflows. We change the $w$ in PC-RED to make the average dropping rates more or less sensitive, and study how it impacts the throughput. Finally, we simulate networks with $10 \%$, and $30 \%$ high-priority dataflows separately to exam the effect of $r_{p}$.

\section{Experiment 1}

We set a network with 20 dataflows, 100ms delay, link capacity 125 packets/s, and the router parameters as follow: $p_{\max }=0.1, \max _{t h}=35, \min _{t h}=10$, Buffer $=50$, $\alpha=4 \mathrm{e}-3$. We set dataflow $i$ to be the only dataflow with priority in the network. PC-RED parameters are: $N_{p}=1$, $w=3 e-3$.

To compare PC-RED with RED, the average queue size and the throughput of one of the dataflows when the router using RED and PC-RED are shown in Table I.

TABLE I

COMPARISON OF RED AND PC-RED

\begin{tabular}{ccccccc}
\hline \multirow{2}{*}{ AQM } & $\begin{array}{c}\text { Average } \\
\text { Queue Size }\end{array}$ & \multirow{2}{*}{$\begin{array}{c}\text { Fluctuation } \\
\text { (packets) }\end{array}$} & \multicolumn{3}{c}{ Throughput (KB/s) } \\
\cline { 4 - 6 } & 33 & 2 & 1.8 & 5.9 & 3.85 \\
\hline RED & 33 & 5 & 5 & 18 & 11.5 \\
PC- & 33 & min & max & average \\
RED & & &
\end{tabular}

The queue length of RED and PC-RED are both stable. However, due to the extra operating time caused by the PCRED Priority List Maintenance Function and the special Pick Packet to Drop Mechanism, the fluctuation of queue length in PC-RED is larger than the one in RED. The throughput of high-priority dataflow increases to nearly 3 times of it in RED. Comprehensively considering the queue length and the throughput, the PC-RED performance is very satisfying.

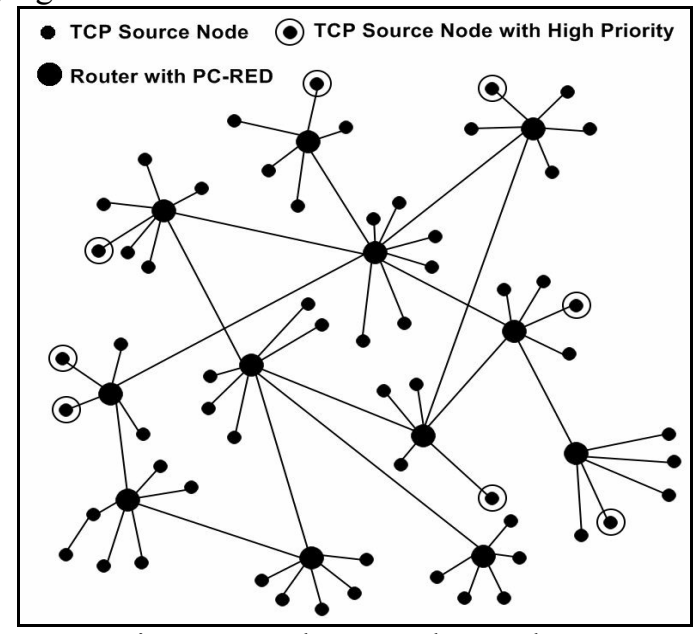

\section{Experiment 2}

Figure 5 Mash Network Topology

In Network 1, we set $w=5 e-3$ and $w=3 e-5$, then compare $a_{d}$ and the throughput of one node under these two conditions in Table II. The desired limit $L$ of high-priority dataflow is converted into size and set to be 70 bytes per second and the simulation duration is 3000 seconds throughout the following simulations in the rest of the paper.

When $w$ is large, the $a_{d}$ is sensitive to the dropping action. This guarantees a certain level of quality of service to highpriority dataflow, and ensures a higher rank of throughput. When $w$ is small, the $a_{d}$ responses tardily to the dropping action. And the PC-RED cannot help the high-priority dataflow to achieve a satisfying throughput in a long scale of time as well. 
TABLE II

COMPARISON OF DIFFERENT $w$ IN NETWORK1

\begin{tabular}{cccccc}
\hline \multirow{2}{*}{$w$} & $a_{d}$ & Fluctuation & \multicolumn{3}{c}{ Throughput (KB/s) } \\
\cline { 4 - 6 } & (bytes/s) & (bytes/s) & min & max & average \\
\hline $5 \mathrm{e}-3$ & 71 & 8 & 3 & 14 & 8.5 \\
$3 \mathrm{e}-3$ & $\begin{array}{c}\text { Under } L \text { until } \\
2700 \mathrm{~s}\end{array}$ & - & 0 & 5 & 2.5 \\
\hline
\end{tabular}

Network 2 consists of 480 source nodes and 12 routers. We compare the throughput of the same node when $w$ is set to $5 \mathrm{e}-$ 3 and $3 \mathrm{e}-3$ in Table III. The result also shows that the higher $w$ can help the source to achieve a better throughput.

TABLE III

COMPARISON OF DIFFERENT $w$ IN NETWORK2

\begin{tabular}{cccc}
\hline \multirow{2}{*}{$w$} & \multicolumn{3}{c}{ Throughput (KB/s) } \\
\cline { 2 - 4 } & $\min$ & $\max$ & average \\
\hline $5 \mathrm{e}-3$ & 6.2 & 9 & 7.6 \\
$3 \mathrm{e}-3$ & 2 & 5.2 & 3.5 \\
\hline
\end{tabular}

\section{Experiment 3}

The comparison between the throughput of the high-priority dataflow and the throughput of the non-priority dataflow in Network 1 is shown in Table IV when $r_{p}$ is $10 \%$ and $30 \%$. In this simulation we set $w=e-3$.

\section{TABLE IV}

COMPARISON OF DIFFERENT $r_{p}$ IN NETWORK1

\begin{tabular}{ccccccc}
\hline \multirow{2}{*}{$r_{p}$} & \multirow{2}{*}{ Priority } & $a_{d}$ & Fluctuation & \multicolumn{3}{c}{$\begin{array}{c}\text { Throughput } \\
\text { (KB/s) }\end{array}$} \\
\cline { 5 - 7 } & & (bytes/s) & (bytes/s) & min & max & ave \\
\hline $10 \%$ & High & 70 & 2 & 2.5 & 19 & 10.8 \\
$10 \%$ & Non & 120 & 23 & 0 & 6 & 3 \\
$30 \%$ & High & 80 & 4 & 4 & 12 & 8 \\
$30 \%$ & Non & 130 & 45 & 0 & 1 & 0.5 \\
\hline
\end{tabular}

When $r_{p}$ is $10 \%$, the throughput of high-priority dataflow is about 3.2 times as the throughput of non-priority dataflow. And $a_{d}$ is under $\mathrm{L}$ as well. When $r_{p}$ rises up to $30 \%$, since the ratio of $N_{p}$ to $N$ is larger, according to (4), the dropping probability of the high-priority dataflow would also increase. Based on (5) and (6), the system has reached its critical state due to the large $r_{p}$. Although the PCRED priority checking function has been enabled, it is still unable to control the $a_{d}$ of high-priority dataflow under L. And the $a_{d}$ of non-priority dataflow oscillates more acutely than previously, which leads to an extremely low level of throughput. In addition, the throughput of high-priority dataflow is also lower than it was in a network with smaller amount of high-priority dataflows. TABLE $\mathrm{V}$

COMPARISON OF DIFFERENT $r_{p}$ IN NETWORK2

\begin{tabular}{ccccc}
\hline \multirow{2}{*}{$r_{p}$} & Priority & \multicolumn{3}{c}{ Throughput (KB/s) } \\
\cline { 3 - 5 } & & $\min$ & $\max$ & average \\
\hline $10 \%$ & High & 4.7 & 8.3 & 6.5 \\
$10 \%$ & Non & 1.2 & 5.1 & 3.15 \\
$30 \%$ & High & 5 & 6.8 & 5.9 \\
$30 \%$ & Non & 0 & 4.5 & 2.25 \\
\hline
\end{tabular}

The throughputs of the high-priority node and of the nonpriority node in Network 2 are compared in Table V. The result also shows that a large $r_{p}$ leads to an obvious reduction and a larger oscillation of the throughput.

\section{CONCLUSION}

In this paper a new RED with Priority Checking function is provided as an AQM method of a network supporting both high-priority dataflows and non-priority dataflows. The Priority Checking function does not involve the traditional RED parameters. But the extra function process times do affect the queue status and slightly increase the fluctuation when the performance is still stable. The NS2 simulation shows that the PCRED has guaranteed a certain level of the average dropping rate of high-priority dataflows and helped the data source to achieve desirable transient performance. The influences of the average dropping rate weight factor and the percentage of high-priority dataflows in overall load level have been studied. How to guarantee the transient performance of non-priority dataflows in a network supporting PCRED and the implementation of such AQM method with Priority-Checking function in a wider range of networks such as multi-switched and wireless network and the further research on the design of the advanced packet scheduling mechanism in PC-RED would be studied in the future.

\section{ACKNOWLEDGEMENTS}

Thanks to Loughborough University Computer Science Department for sponsoring and to Fang YAO, Khusvinder GILL, and Wu CHEN for their help and inspiration.

\section{REFERENCES}

[1] L. Le, J. Aikat, K. Jeffay and F. D. Smith, The effect of active queue management on Web performance, in Proceedings of SIGCOMM'03, Karlsruhe, Germany, Aug. 2003, pp. 265-276.

[2] M. May, J. Bolot, C. Diot and B. Lyles, Reasons not to deploy RED, in Proceedings of 7th International Workshop on Quality of Service, London, 31 May-4 June 1999, pp. 260-262.

[3] S. Floyd, R. Gummadi and S. Shenker, Adaptive RED: an algorithm for increasing the robustness of RED's active queue management. [Online] Available: http://www.icir.org/floyd/papers/adaptiveRed.pdf, Aug. 2001.

[4] W. CHEN, Y. LI, S.H. YANG, An Average Queue Weight Parameterization in a Network Supporting TCP with RED, IEEE ICNSC, 2007

[5] Zhang, L., Zheng, L., IPv6 traffic with multi-class QoS in VPN, COMPUTER NETWORKS -AMSTERDAM- 2001 ; VOL 37; NUMBER 3-4 ; Pages: 263-275

[6] V. Misra, W.B. Gong, and D. Towsley, Fluid-based Analysis of a Network of AQM Routers Supporting TCP Flows with an Application to RED, in Proceedings of ACM/SIGCOMM, 2000.

[7] C. Hollot, V. Misra, D. Towlsey, and W. Gong, A control theoretic analysis of red, in the proceedings of IEEE Infocom 2001, Anchorage,Alaska, Apr. 2001. 